\title{
Continuous solutions of a polynomial-like iterative equation with variable coefficients
}

\author{
by Weinian Zhang (Chengdu) and John A. Baker (Waterloo, ON)
}

\begin{abstract}
Using the fixed point theorems of Banach and Schauder we discuss the existence, uniqueness and stability of continuous solutions of a polynomial-like iterative equation with variable coefficients.
\end{abstract}

I. Introduction. Let $I=[a, b]$ be a given closed bounded interval. Given a continuous $F: I \rightarrow I$ such that $F(a)=a$ and $F(b)=b$, and given continuous functions $\lambda_{1}, \ldots, \lambda_{n}: I \rightarrow[0,1]$ such that $\sum_{i=1}^{n} \lambda_{i}(x)=1$ for all $x \in I$, we wish to find continuous functions $f: I \rightarrow I$ such that

(1) $\quad \lambda_{1}(x) f(x)+\lambda_{2}(x) f^{2}(x)+\ldots+\lambda_{n}(x) f^{n}(x)=F(x) \quad$ for all $x \in I$.

Here $f^{i}$ denotes the $i$ th iterate of $f$ (i.e., $f^{0}(x)=x$ and $f^{i+1}(x)=f\left(f^{i}(x)\right)$ for all $x \in I$ and all $i=0,1, \ldots)$. We suppose that $n \geq 2$.

The case in which the $\lambda_{i}$ 's are constant was considered in [4]-[7] and [9]-[11] for special choices of $F$ and/or $n$. Similar equations are discussed on pages 237-240 of [5]. Such problems are related both to problems concerning iterative roots (see [1], [3] and [8]), e.g. finding a function $f$ such that

$$
f^{n}(x)=F(x), \quad \forall x \in I,
$$

and to the theory of invariant curves for mappings (see Chapter XI of [5]).

Note that we may assume without loss of generality that $a=0$ and $b=1$. Indeed, if $[a, b] \neq[0,1]$ and (1) holds, define

$$
h(t)=a+t(b-a) \quad \text { for } 0 \leq t \leq 1
$$

and let

$$
g=h^{-1} \circ f \circ h, \quad G=h^{-1} \circ F \circ h, \quad \mu_{i}=\lambda_{i} \circ h \quad \text { for } 1 \leq i \leq n
$$

2000 Mathematics Subject Classification: 39B12, 47H99.

Key words and phrases: functional equation, iterative root, fixed point theorem.

The second author supported by NSERC (Canada) Grant \#7153. 
where $\circ$ denotes composition. Since $h$ and $h^{-1}$ are affine and $\sum_{i=1}^{n} \lambda_{i}(x)=1$ for all $x \in I$, it follows that

$$
\sum_{i=1}^{n} \mu_{i}(t) g^{i}(t)=G(t) \quad \text { for all } t \in[0,1] .
$$

Conversely, if (2) holds so does (1). Thus assume that $I=[0,1]$.

For economy of exposition we adopt the following notation. Let $C(I)$ denote the real Banach algebra consisting of all continuous maps of $I$ into $\mathbb{R}$ with respect to the uniform norm; for $f \in C(I),\|f\|=\max \{|f(t)|: t \in I\}$. Let

$$
X=\{f \in C(I): 0=f(0) \leq f(t) \leq f(1)=1 \text { for all } t \in I\} .
$$

Note that $X$ is closed under composition and hence under iteration.

For $0 \leq m \leq 1 \leq M$ let

$$
\begin{array}{r}
X(m, M)=\{f \in X: m(y-x) \leq f(y)-f(x) \leq M(y-x) \\
\text { whenever } 0 \leq x \leq y \leq 1\} .
\end{array}
$$

\section{Some lemmas}

Lemma 1. Suppose $0 \leq m \leq 1 \leq M$. Then $X(m, M)$ is a compact convex subset of $C(I)$. Moreover, if $f, g \in X(m, M)$ then

$$
\left\|f^{\nu}-g^{\nu}\right\| \leq \sum_{j=0}^{\nu-1} M^{j}\|f-g\| \quad \text { for all } \nu=1,2, \ldots
$$

Pr o of. It is clear that $X(m, M)$ is a closed, bounded and convex subset of $C(I)$. It is also clear that $X(m, M)$ is uniformly equicontinuous. Thus, by the Ascoli-Arzelà lemma, $X(m, M)$ is a compact convex subset of $C(I)$.

If $\nu=1$ then the inequality is trivial. Suppose it holds when $1 \leq \nu \leq k$ for some $k \geq 1$. Then, for all $x \in I$,

$$
\begin{aligned}
\left|f^{k+1}(x)-g^{k+1}(x)\right| & =\left|f\left(f^{k}(x)\right)-g\left(g^{k}(x)\right)\right| \\
& \leq\left|f\left(f^{k}(x)\right)-f\left(g^{k}(x)\right)\right|+\left|f\left(g^{k}(x)\right)-g\left(g^{k}(x)\right)\right| \\
& \leq M\left\|f^{k}-g^{k}\right\|+\|f-g\| \\
& \leq M\left(\sum_{j=0}^{k-1} M^{j}\right)\|f-g\|+\|f-g\| \\
& =\left(\sum_{j=0}^{k} M^{j}\right)\|f-g\| .
\end{aligned}
$$

Thus, by induction, the inequality is true for all $\nu \geq 1$. 
Lemma 2. Suppose $0<m \leq 1 \leq M$ and $f, g \in X(m, M)$. Then

(i) $f^{-1} \in X\left(M^{-1}, m^{-1}\right)$,

(ii) $\|f-g\| \leq M\left\|f^{-1}-g^{-1}\right\|$, and

(iii) $\left\|f^{-1}-g^{-1}\right\| \leq m^{-1}\|f-g\|$.

Pr o of. Since $m>0, f$ is a strictly increasing homeomorphism of $I$ onto itself and, for $0 \leq x<y \leq 1$,

$$
M^{-1} \leq \frac{y^{\prime}-x^{\prime}}{f\left(y^{\prime}\right)-f\left(x^{\prime}\right)} \leq m^{-1}
$$

where $y^{\prime}=f^{-1}(y)$ and $x^{\prime}=f^{-1}(x)$. Thus (i) holds.

To prove (ii) note that for all $x \in I$,

$$
\begin{aligned}
|f(x)-g(x)| & =\left|f(x)-f\left(\left(f^{-1} \circ g\right)(x)\right)\right| \leq M\left|x-f^{-1}(g(x))\right| \\
& =M\left|g^{-1}(g(x))-f^{-1}(g(x))\right| \leq M\left\|g^{-1}-f^{-1}\right\| .
\end{aligned}
$$

It follows that $\|f-g\| \leq M\left\|g^{-1}-f^{-1}\right\|=M\left\|f^{-1}-g^{-1}\right\|$.

Property (iii) follows easily from (i) and (ii).

These lemmas are essentially Lemmas 2.2 and 2.5 of [11]. Also note that, by (iii), the inversion map $\mathcal{I}: X(m, M) \rightarrow X\left(M^{-1}, m^{-1}\right)$ (defined by $\mathcal{I} f=f^{-1}$ for $\left.f \in X(m, M)\right)$ is a Lipschitz mapping.

Lemma 3. If $f \in X(m, M)$ and $g \in X(s, S)$ with $0 \leq m \leq 1 \leq M$ and $0 \leq s \leq 1 \leq S$, then $f \circ g \in X(m s, M S)$ and

$$
f^{k} \in X\left(m^{k}, M^{k}\right) \quad \text { for all } k=0,1, \ldots
$$

Proof. It suffices to note that, for $0 \leq x \leq y \leq 1$,

$$
f(g(y))-f(g(x)) \leq M(g(y))-g(x)) \leq M S(y-x)
$$

and, similarly,

$$
f(g(y))-f(g(x)) \geq m s(y-x)
$$

III. Existence. Our main result is the following

Theorem 1. Suppose that $\lambda_{1}(x) \geq c$ for all $x \in I$ and

$$
\operatorname{Lip} \lambda_{k}:=\sup \left\{\frac{\left|\lambda_{k}(y)-\lambda_{k}(x)\right|}{y-x}: 0 \leq x<y \leq 1\right\} \leq \beta \quad \text { for } k=1,2, \ldots
$$

where $c$ and $\beta$ are real constants such that

$$
0<c<1 \quad \text { and } \quad 0 \leq n \beta \leq 1 .
$$

Also suppose that $F \in X(\delta, M)$ with

$$
n \beta \leq \delta \leq 1 \leq M .
$$

Then (1) has a solution $f$ in $X(0,(M+n \beta) / c)$. 
Proof. Let $L=(M+n \beta) / c$ and note that $L>1$ since $0<c<1 \leq M$.

For $x \in I$ and $f \in X(0, L)$ define $f_{x}: I \rightarrow \mathbb{R}$ by

$$
f_{x}(t)=\sum_{i=1}^{n} \lambda_{i}(x) f^{i-1}(t) \quad \text { for } t \in I .
$$

Our task is to prove that, for some $f \in X(0, L)$,

$$
f_{x}(f(x))=F(x) \quad \text { for all } x \in I .
$$

The idea behind our proof is based on the observation that if every $f_{x}$ were a bijection of $I$ then $(1)^{\prime}$ would be equivalent to

$$
f(x)=\left(f_{x}\right)^{-1}(F(x)) \quad \text { for all } x \in I ;
$$

i.e. recasting the problem as a fixed point problem.

Suppose $f \in X(0, L)$ and $x \in I$. Then $f_{x}(0)=0, f_{x}(1)=1, f_{x}(t) \in I$ for all $t \in I$ and $f_{x}$ is continuous. Moreover, if $0 \leq t \leq u \leq 1$ then, by Lemma 3 ,

$$
\begin{aligned}
f_{x}(u)-f_{x}(t) & =\sum_{i=1}^{n} \lambda_{i}(x)\left(f^{i-1}(u)-f^{i-1}(t)\right) \\
& \leq \sum_{i=1}^{n} \lambda_{i}(x) L^{i-1}(u-t) \leq\left(\sum_{i=1}^{n} L^{i-1}\right)(u-t)
\end{aligned}
$$

and

Thus

$$
f_{x}(u)-f_{x}(t) \geq \lambda_{1}(x)(u-t) \geq c(u-t)
$$

$$
f_{x} \in X(c, C) \quad \text { for } x \in I \text { and } f \in X(0, L)
$$

where $C=\sum_{i=1}^{n} L^{i-1}$.

If $f \in X(0, L), 0 \leq x<y \leq 1$ and $t \in I$ then

$$
\left|f_{y}(t)-f_{x}(t)\right|=\left|\sum_{i=1}^{n}\left(\lambda_{i}(y)-\lambda_{i}(x)\right) f^{i-1}(t)\right| \leq n \beta(y-x) .
$$

Thus

$$
\left\|f_{y}-f_{x}\right\| \leq n \beta|y-x| \quad \text { for } f \in(0, L) \text { and } x, y \in I .
$$

Now suppose that $f \in X(0, L), 0 \leq x<y \leq 1$ and $t \in I$. By (3) and (4),

$$
\begin{aligned}
0=t-t & =f_{y}\left(f_{y}^{-1}(t)\right)-f_{x}\left(f_{x}^{-1}(t)\right) \\
& =f_{y}\left(f_{y}^{-1}(t)\right)-f_{y}\left(f_{x}^{-1}(t)\right)+f_{y}\left(f_{x}^{-1}(t)\right)-f_{x}\left(f_{x}^{-1}(t)\right) \\
& \geq c\left(f_{y}^{-1}(t)-f_{x}^{-1}(t)\right)-n \beta(y-x)
\end{aligned}
$$

and, similarly,

so that

$$
0 \leq C\left(f_{y}^{-1}(t)-f_{x}^{-1}(t)\right)+n \beta(y-x)
$$

$$
-n \beta C^{-1} \leq\left(f_{y}^{-1}(t)-f_{x}^{-1}(t)\right) /(y-x) \leq n \beta c^{-1} .
$$


Thus, for $f \in X(0, L)$,

$$
\left\|f_{y}^{-1}-f_{x}^{-1}\right\| \leq n \beta c^{-1}|y-x| \quad \text { for all } x, y \in I
$$

since $0<c<1<L<C$.

Now for $f \in X(0, L)$ define $T f: I \rightarrow \mathbb{R}$ by

$$
T f(x)=f_{x}^{-1}(F(x)) \quad \text { for } x \in I
$$

notice that $T f(0)=0, T f(1)=1$ and $T f(x) \in I$ for all $x \in I$.

Suppose that $f \in X(0, L)$ and $0 \leq x<y \leq 1$. By (5) and (i) of Lemma 2,

$$
\begin{aligned}
T f(y)-T f(x) & =f_{y}^{-1}(F(y))-f_{x}^{-1}(F(x)) \\
& =f_{y}^{-1}(F(y))-f_{x}^{-1}(F(y))+f_{x}^{-1}(F(y))-f_{x}^{-1}(F(x)) \\
& \leq n \beta c^{-1}(y-x)+c^{-1}(F(y)-F(x)) \\
& \leq(n \beta+M) c^{-1}(y-x)=L(y-x) .
\end{aligned}
$$

Similarly,

$$
\begin{aligned}
T f(y)-T f(x) & =f_{y}^{-1}(F(y))-f_{x}^{-1}(F(y))+f_{x}^{-1}(F(y))-f_{x}^{-1}(F(x)) \\
& \geq\left(-n \beta C^{-1}\right)(y-x)+C^{-1}(F(y)-F(x)) \\
& \geq(-n \beta+\delta) C^{-1}(y-x) \geq 0
\end{aligned}
$$

since $n \beta \leq \delta \leq 1$. Thus $T f \in X(0, L)$. We conclude that $T$ maps $X(0, L)$ into itself.

Aiming to prove that $T$ is continuous, suppose that $f, g \in X(0, L)$. By the lemmas, for any $x \in I$ we have

$$
\begin{aligned}
|T f(x)-T g(x)| & =\left|f_{x}^{-1}(F(x))-g_{x}^{-1}(F(x))\right| \leq\left\|f_{x}^{-1}-g_{x}^{-1}\right\| \\
& \leq c^{-1}\left\|f_{x}-g_{x}\right\| \leq c^{-1} \max _{t \in I} \sum_{i=2}^{n} \lambda_{i}(x)\left|f^{i-1}(t)-g^{i-1}(t)\right| \\
& \leq c^{-1} \sum_{i=2}^{n} \lambda_{i}(x)\left\|f^{i-1}-g^{i-1}\right\| \\
& \leq c^{-1} \sum_{i=2}^{n} \lambda_{i}(x)\left(\sum_{j=0}^{i-2} L^{j}\right)\|f-g\| \\
& \leq c^{-1}\left(\sum_{j=0}^{n-2} L^{j}\right)\left(\sum_{i=2}^{n} \lambda_{i}(x)\right)\|f-g\| \\
& =c^{-1}\left(\sum_{j=0}^{n-2} L^{j}\right)\left(1-\lambda_{1}(x)\right)\|f-g\| \\
& \leq c^{-1}(1-c)\left(\sum_{j=0}^{n-2} L^{i}\right)\|f-g\|
\end{aligned}
$$


recall that $0<c<1$ and $c \leq \lambda_{1}(x)$ for all $x \in I$. We have proved that

$$
\|T f-T g\| \leq \gamma\|f-g\| \quad \text { for all } f, g \in X(0, L)
$$

where

$$
\gamma=c^{-1}(1-c) \sum_{j=0}^{n-2} L^{j} .
$$

Thus $T$ is continuous. By Schauder's fixed point theorem $T$ has a fixed point, i.e., $(1)^{\prime \prime}$ holds for some $f \in X(0, L)$.

IV. Uniqueness and stability. If $\gamma<1$ then $T$ is a contraction, in which case Banach's fixed point theorem implies that our problem has a unique solution.

THEOREM 2. If, in addition to the assumptions of Theorem 1, $c$ is so close to 1 that

$$
(1-c) \sum_{j=1}^{n-1}(M+n \beta)^{j-1} / c^{j}<1
$$

then (1) has a unique solution $f$ in $X(0,(M+n \beta) / c)$.

Proof. It suffices to note (7) and (8) and recall that $L=(M+n \beta) / c$.

Under the assumptions of Theorem 2, the solution to our problem depends continuously upon the given data in the sense of

THEOREM 3. In addition to the assumptions of Theorem 2 , suppose that $\mu_{1}, \ldots, \mu_{n}: I \rightarrow I$ are continuous, $\sum_{i=1}^{n} \mu_{i}(x)=1$ for all $x \in I, \mu_{1}(x) \geq c$ for all $x \in I$,

$$
\left|\mu_{k}(y)-\mu_{k}(x)\right| \leq \beta|y-x| \quad \text { for } x, y \in I \text { and } 1 \leq k \leq n
$$

and $G \in X(\delta, M)$. Let $g$ be that member of $X(0, L)$ satisfying

$$
\sum_{k=1}^{n} \mu_{k}(x) g^{k}(x)=G(x) \quad \text { for all } x \in I
$$

(whose existence and uniqueness is guaranteed by Theorem 2). Then

$$
\|f-g\| \leq(1-\gamma)^{-1} c^{-1}\left(\sum_{i=1}^{n}\left\|\lambda_{i}-\mu_{i}\right\|+\|F-G\|\right) .
$$

Proof. To indicate the dependence of the relevant operators on the given data, let us write $\lambda_{x} \varphi$ instead of $\varphi_{x}$ for $\varphi \in X(0, L)$ and write $T_{\lambda}$ instead of $T$. For $\varphi \in X(0, L)$ and $x \in I$ define $\mu_{x} \varphi(t)=\sum_{i=1}^{n} \mu_{i}(x) \varphi^{i-1}(t)$ for $t \in I$. For $\varphi \in X(0, L)$ let

$$
T_{\mu} \varphi(x)=\left(\mu_{x} \varphi\right)^{-1}(G(x)) \quad \text { for } x \in I .
$$


Suppose then that $f, g \in X(0, L),(1)$ and (9) hold and $x \in I$. Then

$$
\begin{aligned}
|f(x)-g(x)|= & \left|\left(\lambda_{x} f\right)^{-1}(F(x))-\left(\mu_{x} g\right)^{-1}(G(x))\right| \\
\leq & \left|\left(\lambda_{x} f\right)^{-1}(F(x))-\left(\mu_{x} g\right)^{-1}(F(x))\right| \\
& +\left|\left(\mu_{x} g\right)^{-1}(F(x))-\left(\mu_{x} g\right)^{-1}(G(x))\right| \\
\leq & \left\|\left(\lambda_{x} f\right)^{-1}-\left(\mu_{x} g\right)^{-1}\right\|+c^{-1}|F(x)-G(x)| \\
\leq & c^{-1}\left\{\left\|\lambda_{x} f-\mu_{x} g\right\|+\|F-G\|\right\}
\end{aligned}
$$

by Lemma 2 since $\lambda_{x} f, \mu_{x} g \in X(c, C)$. By using Lemma 1 several times we find that, for all $t \in I$,

$$
\begin{aligned}
\left|\lambda_{x} f(t)-\mu_{x} g(t)\right| & =\left|\sum_{i=1}^{n} \lambda_{i}(x) f^{i-1}(t)-\mu_{i}(x) g^{i-1}(t)\right| \\
& \leq \sum_{i=1}^{n}\left|\lambda_{i}(x)-\mu_{i}(x)\right|\left|f^{i-1}(t)\right|+\sum_{i=1}^{u} \mu_{i}(x)\left|f^{i-1}(t)-g^{i-1}(t)\right| \\
& \leq \sum_{i=1}^{n}\left\|\lambda_{i}-\mu_{i}\right\|+\sum_{i=2}^{n} \mu_{i}(x)\left\|f^{i-1}-g^{i-1}\right\| \\
& \leq \sum_{i=1}^{n}\left\|\lambda_{i}-\mu_{i}\right\|+\sum_{i=2}^{n} \mu_{i}(x)\left(\sum_{j=0}^{i-2} L^{j}\right)\|f-g\| \\
& \leq \sum_{i=1}^{n}\left\|\lambda_{i}-\mu_{i}\right\|+\sum_{i=2}^{n} \mu_{i}(x)\left(\sum_{j=0}^{n-2} L^{j}\right)\|f-g\| \\
& =\sum_{i=1}^{n}\left\|\lambda_{i}-\mu_{i}\right\|+\left(1-\mu_{1}(x)\right) c(1-c)^{-1} \gamma\|f-g\| \\
& \leq \sum_{i=1}^{n}\left\|\lambda_{i}-\mu_{i}\right\|+(1-c) \gamma c(1-c)^{-1}\|f-g\|
\end{aligned}
$$

by the definition (8) of $\gamma$. It follows that

$$
\|f-g\| \leq c^{-1}\left\{\sum_{i=1}^{n}\left\|\lambda_{i}-\mu_{i}\right\|+\gamma c\|f-g\|+\|F-G\|\right\},
$$

i.e., (10) holds.

V. Remarks and questions. The normalization assumption that $\sum_{i=1}^{n} \lambda_{i}(x)=1$ is not severe. Instead one could suppose that $\lambda_{i}: I \rightarrow[0, \infty)$ is continuous for $1 \leq i \leq n$ and $\sum_{i=1}^{n} \lambda_{i}(x)>0$ for all $x \in I$. Then the equation can be normalized by dividing by $\sum_{i=1}^{n} \lambda_{i}(x)$; of course, the assumptions on $F$ would have to be altered appropriately. 
We conclude the paper with some questions for possible future discussion.

1. How can (1) be treated without the assumption that $\lambda_{1}(x) \geq c>0$ for all $x \in I$ ?

2. What more can be said in case the given functions $\lambda_{1}, \ldots, \lambda_{n}$ and $F$ are smooth?

3. What can be said in case $F(0)=1$ and $F(1)=0$ ?

\section{References}

[1] N. H. Abel, Oeuvres complètes, Vol. II, Christiania, 1981, 36-39.

[2] J. G. Dhombres, Itération linéaire d'ordre deux, Publ. Math. Debrecen 24 (1977), $277-287$.

[3] J. M. Dubbey, The Mathematical Work of Charles Babbage, Cambridge Univ. Press, 1978.

[4] M. Kuczma, Functional Equations in a Single Variable, Monograf. Mat. 46, PWN, Warszawa, 1968

[5] M. Kuczma, B. Choczewski, and R. Ger, Iterative Functional Equations, Encyclopedia Math. Appl. 32, Cambridge Univ. Press, 1990.

[6] A. Mukherjea and J. S. Ratti, On a functional equation involving iterates of a bijection on the unit interval, Nonlinear Anal. 7, (1983), 899-908.

[7] S. Nabeya, On the function equation $f(p+q x+r f(x))=a+b x+c f(x)$, Aequationes Math. 11 (1974), 199-211.

[8] J. Z. Zhang and L. Yang, Discussion on iterative roots of continuous and piecewise monotone functions, Acta Math. Sinica 26 (1983), 398-412 (in Chinese).

[9] W. N. Zhang, Discussion on the iterated equation $\sum_{i=1}^{n} \lambda_{i} f^{i}(x)=F(x)$, Chinese Sci. Bull. 32 (1987), 1444-1451.

[10] - Stability of the solution of the iterated equation $\sum_{i=1}^{n} \lambda_{i} f^{i}(x)=F(x)$, Acta Math. Sci. 8 (1988), 421-424.

[11] - Discussion on the differentiable solutions of the iterated equation $\sum_{i=1}^{n} \lambda_{i} f^{i}(x)$ $=F(x)$, Nonlinear Anal. 15 (1990), 387-398.

Department of Mathematics

Sichuan Union University

Chengdu 610064

P.R. China
Department of Pure Mathematics University of Waterloo Waterloo, Ontario, Canada N2L 3G1

E-mail: jabaker@math.uwaterloo.ca 